\title{
Review
}

\section{Review of Research on Immunotherapy of Severe Surgical Infection}

\author{
Quan Liang
}

Department of General Surgery, Tianjin Medical University General Hospital, Tianjin China

\author{
Keywords \\ severe surgical infection; immune nutrition; \\ immune regulation

\section{Correspondence} \\ Quan Liang, \\ E-mail: liangq01@126.com \\ DOI: 10.1515/ii-2017-0122
}

\begin{abstract}
Severe surgical infection refers to infection after surgical treatment or surgical operation, accounting for approximately $30 \%$ of surgical diseases. This type of infection can cause extensive inflammation and tissue injury and reduce success rate of surgical treatments. Immune defense plays an important role in antiinfection treatment of hosts. Infection immunity is a series of physiological defense mechanisms for recognition of immune system and removal of pathogens. As for severe surgical infection, immunotherapy becomes an important and promising therapy for severe surgical infection. This study summarizes recent progresses achieved in immunization and immunotherapy of surgical infection.
\end{abstract}

\section{Immune mechanism of severe surgical infection} Immunological characteristics and changes in severe
surgical infection

Infectious immune responses include innate immunity and adaptive immunity. Innate immunity includes barrier immunity, complement replacement pathway, phagocytic cells, cytokines, and natural killer (NK) cells. Adaptive immunity includes CD4+ cell functional differentiation, cellular immune response, humoral immune response, and immune memory. Immune and adaptive immunity against pathogens feature the following characteristics: (1) inherent immune functions in preinfection, adaptive immune response functions in late infection; these functions promote and complement each other; (2) different antiinfection immune mechanisms induced by different types of pathogens; (3) pathogens can escape and resist immune response, especially for inherent immune response with poor specificity; and (4) injury and disease caused by infection not only result from pathogens but also from inappropriate immune response of the body itself.

Followed by severe surgical infection, adaptive immune response changes, mainly affecting $\mathrm{T}$ helper (Th) cells and temporarily inhibiting Thl-type cytokine-mediated pathway but not affecting Th2-type cytokine reaction, leading to imbalance of Thl/Th2 ratio of major surgeries. Levels of antiinflammatory cytokines significantly increase in severe surgical infections. T cells shift from Th1 to Th2, resulting in immune disorders or immunosuppression. Surgical infection can cause systemic inflammatory response and severe immunosuppression, leading to sepsis. Sepsis can also lead to immunosuppression, which is characterized by reduction of mononuclear cells expressing human leukocyte antigen (HLA), damaged capability of mononuclear cells that stimulate $\mathrm{T}$ cells, decrease in host antibacterial virus-related Th1 cytokines, and increase in host's immunosuppressionrelated Th2 cytokines and regulatory T-cell factor [interleukin (IL)-4 and IL-10]. Sepsis is associated with significant intrinsic and adaptive immune dysfunctions. Immune imbalance is mainly manifested as dysfunctions of cellular immune and immunoregulatory cells, leading to immune paralysis.

\section{Influence of surgical trauma on immune system}

Surgical trauma displays different effects on both innate immune cells and adaptive immune cells. Antigenic products resulting from tissue injury can activate intrinsic immune cells to secrete various inflammatory mediators. After activation of innate immune cells, together with molecules 
on $\mathrm{T}$ cell receptor, various stimulating molecules initiate adaptive immune responses. Affected by cytokines and antigens, Th cells differentiate into Thl and IL-2 subsets. IL-2 and interferon (IFN) $-\gamma$ secreted by Thl may promote cell-mediated immune response. IL-4 and IL-10 secreted by Th2 can enhance antibody-mediated immune response. Tissue injury may initially cause innate immune response through macrophages, NK cells, and neutrophils. Adaptive immune response is initiated by presentation of exogenous antigens to CD4+ T cells and CD8+ T cells. Initiated CD4+ $\mathrm{T}$ cells can secrete cytokines to amplify immune response, in which Th1 cells secrete proinflammatory cytokines, and Th2 cells secrete antiinflammatory cytokines. Accordingly, monitoring Th1 and Th2 cytokine expression levels or their cytokines may reflect in vivo inflammation. Surgery can sensitize host immune systems. Then, subsequent relatively slight attacks can induce severe host inflammatory and immune responses, further leading to multiple organ failure and death. This phenomenon is called the second attack caused by surgery, and it also increases incidence of surgical infection.

Surgical trauma can induce the immune system to rapidly produce proinflammatory cytokines and other inflammatory mediators. Antiinflammatory and proinflammatory responses concurrently occur in the body. When proinflammatory response is more significant than antiinflammatory response, systemic inflammatory response syndrome (SIRS) and inflammatory mediator cascade occur. Initiation of reticuloendothelial system may result in increased telangiectasia and permeability, water accumulation of the third gap, and severe shock. Followed by initial inflammatory response, compensatory resistance of antiinflammatory response is generated. When antiinflammatory response is more significant than proinflammatory response, compensatory antiinflammatory response syndrome (CARS) occurs, and antiinflammatory cytokines exhibit strong immunosuppressive effects. At this point, the body's susceptibility to pathogenic microorganisms increases and becomes prone to causing severe postoperative infection complications. SIRS and CARS represent immune hyperactivity or inhibition caused by breakdown of immune self-steady state. Uncontrolled inflammatory response eventually leads to multiple organ dysfunctions.

\section{Immunodeficiency support for severe surgical infection}

\section{Immune nutrition}

Surgical nutrition support can improve organic metabolism and correct negative nitrogen balance, which is conducive to tissue repair, organ maintenance, and patient rehabilitation. Surgical nutrition support can also provide material basis for synthesis of immune proteins, which are conducive to generation of a variety of immune factors and media. Some nutrients, such as ammonia acid, glutamine, and fish oil, may be directly involved in immune function regulation. Nutritional support with glutamine can maintain intestinal barrier function and regulate immune functions. The intestinal tract is also an important immune organ, covering $60 \%$ of lymphocytes. Intestinal barrier dysfunction can lead to intestinal endotoxin and bacterial translocation and then generate SIRS, sepsis, and multiple organ dysfunction syndrome. Intestinal barrier functions include intestinal mucosal mechanical barrier, immune barrier of intestinal endocrine IgA lymphatic system, chemical barrier composed by gastrointestinal digestive juice, and biological barrier composed by intestinal normal bacteria.

\section{Application of intestinal immune nutrients}

Amino acids, antioxidant vitamins, minerals, long chain $\mathrm{n}$ -3 fatty acids, and nucleotides can regulate and improve immune functions. Enteral immune nutrition mixtures include arginine, long chain n-3 fatty acids, and nucleotides, which are widely used among operative patients and seriously ill patients. Xiaohua et al. ${ }^{[1]}$ randomly divided 96 cases of gastric cancer patients into enteral immunodeficiency group (study group) and conventional enteral nutrition group (control group). These researchers provided equal nitrogen and equal-calorie enteral nutrition support to patients from second day to eighth day after operation and tested relevant indicators from the first day to ninth day. Nitrogen was also tested, and its balance was calculated within 24 $\mathrm{h}$ of drug administration. Results showed significantly higher prealbumin, IgA, CD4, and CD4/CD8 in the study group than the control group. IL- 6 and tumor necrosis factor (TNF)- $\alpha$ were significantly lower than those in the control group, proving that enteral immune nutrition can reduce postoperative inflammatory response and improve immune functions of patients with gastric cancer. Okamoto et al. ${ }^{[2]}$ divided 60 patients with gastric cancer into two groups. One group was provided with arginine and omega-3 fatty acids, and another group was served 
with standard diet for seven days before operation. Results showed that postoperative infection complication rate of immunodeficiency group reached $6 \%$, which is significantly lower than the $28 \%$ observed in conventional diet group. This result is similar to that in duration of SIRS. From the first day to seventh day before operation, CD4+ T cells of immunodeficiency group were significantly higher than those in the conventional diet group. $\mathrm{CD} 4+\mathrm{T}$ cells may play an important role in postoperative immunoregulation and inflammatory response in patients undergoing gastrectomy. Cerantola et al. ${ }^{[3]}$ conducted a metaanalysis on perioperative immunological effects on gastrointestinal surgery patients, including 2730 patients in 21 randomized controlled trials. Results showed that providing enteral immunonutrition to gastrointestinal surgery patients in perioperative period can reduce postoperative infection morbidity and hospitalization time but exhibits no effect on mortality. Thus, routine application of enteral immunonutrition is recommended to such patients. Zheng et al. ${ }^{[4]}$ carried out a metaanalysis among 1269 gastrointestinal surgery patients in 13 randomized controlled trials. Their results showed that two or more immune nutrients (including arginine, glutamine, and omega-3 fatty acids) can reduce postoperative infection morbidity and hospitalization time. Immune functions can be improved by increasing total number of lymphocytes, CD4+T cells, and IgG level and decreasing IL-6 level.

Intravenous glutamine can increase the proportion of $\mathrm{CD} 4+\mathrm{T}$ cells and $\mathrm{CD} 8+\mathrm{T}$ cells in the blood, improve intestinal barrier function, and reduce occurrence of intestinal bacterial translocation in patients with potential infection. Early studies indicated that glutamine applied among intensive care unit (ICU) patients can reduce mortality compared with parenteral nutrition (PN). Clinical effects of glutamine are related to expression of mononuclear cell HLA D-related (HLA-DR) and production of IFN- $\gamma$ of $\mathrm{T}$ cells. Some clinical reports showed that critically ill patients lack glutamine. Decreased glutamine level in the blood exhibits certain influence on immune function, organ function, and prognosis of patients. Application of parenteral glutamine also poses significant effects. Administering parenteral glutamine in postoperative patients can enhance lymphocyte proliferation in the blood. Parenteral glutamine was provided to abdominal surgery patients for $48 \mathrm{~h}$. Expression of HLA-DR of mononuclear cells showed good level of maintenance. The following are recommendations from the US enteral nutrition mixture application regarding
HLA-DR: (1) may benefit patients with gastrointestinal surgery, burns, and penetrating torso trauma; (2) may benefit some patients with major surgeries, severe head injuries, burns $>30 \%$, and ventilation-dependent nonseptic ICU patients; and (3) may feature no remarkable benefits for ICU patients who are monitored generally and can restore oral intake within five days.

\section{Application of fat emulsion}

Fat emulsion is an important component of PN. Clinically applied fat emulsions mainly comprise soybean oil and/or coconut oil and palm oil. $\mathrm{n}-6$ Polyunsaturated fatty acid (n6 PUFA) or saturated fatty acid (SFA) feature high contents and easily form peroxidation products, resulting in tissue injury and depletion of natural antioxidant storage. In olive oil fat emulsion, high-content PUFA and SFA are replaced by monounsaturated fatty acid (MUFA). Oleic acid ( $\mathrm{n}-9$ MUFA) is the main fatty acid contained in olive oil. Xinying et al. ${ }^{[5]}$ randomly divided 40 critically ill patients into experimental ( $n=19)$ and control ( $n=21)$ groups and provided them with equal nitrogen and equal-heat $\mathrm{PN}$ for seven days. Patients in the control group were treated with long-chain fat emulsion, whereas those in experimental group were treated with olive oil fat emulsion. Effects of PN containing different fatty acids on oxidative stress and inflammatory responses were observed in critically ill patients. Results showed that olive oil fat emulsion reduced excessive inflammatory responses of critically ill patients and oxidative stress injury.

Some clinical studies indicated that fat emulsions containing fish oil can be used in postoperative patients to reduce generation of inflammatory factors (IL-4, TNF- $\alpha$, and IL-6) and protect immune functions (e.g., monocyteexpressed HLA-DR and IFN- $\gamma$ ). Use of fish oil during surgery can reduce the need for mechanical ventilation, reduce rate of reentry in ICU, decrease mortality, and shorten length of hospitalization. Antiinflammatory effects of fish oil on sepsis patients include reduction in white blood cell count, plasma C-reactive protein level, and inflammatory factors generated by endotoxin-stimulated mononuclear cells; increase in stimulated neutrophils generates IL-B5. Marik et al. ${ }^{[6]}$ performed a metaanalysis among 21 randomized prospective controlled trials involving 1918 patients and concluded that preoperative administration of high-risk patients with major surgery administered with arginine and fish oil immunoregulation diet can reduce incidence of postoperative-acquired infection, wound complications, and 
hospitalization time.

\section{Other immunological nutrients}

Antioxidant micronutrients (including zinc, copper, selenium, vitamin $\mathrm{E}$, vitamin $\mathrm{C}$, and $\mathrm{N}$-acetylcysteine) and arginine can increase immune nutrition and improve immune functions and are also gradually applied in clinical treatments.

\section{Immunosuppressive treatment of severe surgical infection}

Thymosin $\alpha 1$ is a protein peptide hormone generated by the thymus, playing an important role in immune response, neuroendocrine regulation, and gene expression. This protein can induce and promote differentiation and maturation of thymocytes, promote transformation of bone marrow stem cells into T lymph (TNF- $\alpha)$, and further differentiate into $\mathrm{T}$ cell subsets with different functions and increase generation of CD4+ and CD8+T. Thymosin $\alpha 1$ can also regulate levels of TNF- $\alpha$, IL-6, IL-10, and other cytokines in severely infected patients, reduce inflammatory responses, and improve immune function of patients. As an immune regulator, thymosin al can delay increase in levels of TNF- $\alpha$, IL-6, and other proinflammatory cytokines when adjusting patients' immune systems, reducing inflammatory response induced by inflammatory mediators, and increasing level of antiinflammatory factor IL-10. Thymosin $\alpha 1$ can also promote the balance between antiinflammatory and proinflammatory factors. Xiaolei randomly divided 45 patients with severe surgical infection into treatment and control groups. Results showed that level of IL-10 increased in the treatment group (using thymosin $\alpha 1$, such as zadaxin). The control group showed significantly high levels of TNF- $\alpha$ and IL-6.

Continuous blood purification (CBP) can regulate immune function disorders, remove some inflammatory factors, improve functions of monocytes and endothelial cells, rebuild immune state of organic immunity, and treat and control serious infections. Jieshou reported 136 cases with severe abdominal infection treated by CBP. Results showed that curative rate of CBP reached $94.8 \%$. According to clinical studies, combined CBP and thymosin $\alpha 1$ can treat severe sepsis and improve cellular immune function, organic function recovery, and prognosis ${ }^{[9]}$.
Ulinastatin is a protease inhibitor that can inhibit multiple enzymes and improve immune function disorder caused by surgical stimuli and abnormal protein metabolism. Ulinastatin can also prevent organ and cell damage caused by surgical stimuli and improve circulation status during shock.

Colony stimulating factor (CSF) is a small molecule polypeptide that regulates replication, proliferation, and differentiation of hematopoietic stem cells. Granulocyte CSF (G-CSF) and granulocyte-macrophage CSF (GMCSF) exhibit positive effects on neutrophils, monocytes, macrophages, and lymphocytes. G-CSF and GM-CSF can delay apoptosis of neutrophils, upgrade expressions of CD11b and CD14 molecules, and enhance adhesion chemotaxis and phagocytosis. Clinical studies demonstrated that G-CSF and GM-CSF can improve mononuclear cell function of severely postoperative infected patients, increase expressions of TNF- $\alpha$ and HLA-DR, increase the number of lymphocytes, regulate $\mathrm{T}$ cell proliferation and differentiation, enhance immune activity of Th1, and reverse low response of adaptive immunity. Thus, these factors can be used to treat granulocyte reduction and surgical infection. Orozco et al. ${ }^{[10]}$ reported 58 cases of nontraumatic abdominal sepsis patients treated by moraxastine (GM-CSF) and antibiotics. Results showed reduced infection complications, hospitalization time, and costs.

\section{Problems and prospects}

Recently, more in-depth studies were performed on immune mechanism and immunotherapy of surgical infection, and several new results were achieved, providing additional immunological theoretical bases and immune therapy means for controlling severe surgical infection. However, few research specifically focused on effects of surgical trauma and infection on immune system. Issues arise from studies of effects and application of immunotherapy. The role of immunomodulator still lacks full clinical evidence. Future surgical immunology research will achieve more feasible results. With development of antibiotics and perioperative sterile technology, serious surgical infection can be controlled effectively.

\section{Declarations}

Acknowledgements 
No.

\section{Competing interests}

The author declares that she has no competing interest.

\section{Authors' contributions}

Q Liang made the literature analysis and wrote, discussed and revised the manuscript of this review.

\section{References}

1 Jiang Xiaohua, Li Ning, Zhu Weiming, etc. The Role of Postoperative Early Enteral Immune Nutrition of Gastric Cancer. Parenteral and Enteral Nutrition, 2005,12(2): 102-104,108.

2 Okamoto Y, Okano K, Izuishi K, et al. Attenuation of the systemic inflammatory response and infectious complications after gastrectomy with preoperative oral arginine and omega-3 fatty acids supplemented immunonutrition. World J Surg, 2009, 33(9): 1815-1821.

3 Cerantola Y, Hubner M, Grass F, et al. Immunonutrition in gastrointestinal surgery. Br J Surg, 2011, 98(1):37-48.

4 Zheng Y, Li F, Qi B, et al. Application of perioperative immunonutrition for gastrointestinal surgery: a meta-analysis of randomized controlled trials. Asia Pac J Clin Nutr, 2007,16 (suppl 1): 253-257.

5 Wang Xinying, Pan Liya, Li Weiqin, et al. N-9 Monounsaturated Fatty Acids Can Reduce Oxidative Stress and Excessive Inflammatory Response in Critically Ill Patients. Parenteral and Enteral Nutrition, 2010, 17 (6): 323 -325.

6 Marik PE, Zaloga GP. Immunonutrition in high-risk surgical patients: a systematic review and analysis of the literature. J Parenter Enteral Nutr,2010,34(4):378-386.

7 Hu Xiaolei. The Effect of Thymosin $\alpha 1$ on the Immune Function and Cytokines of Patients with Severe Surgical Infections. Chinese Journal of Practical Surgery, 2007, 27(7): 555-557.

8 Li Jieshou. Abdominal Infection and Immune Regulation. Chinese Journal of Practical Surgery, 2007, 27(12): 931-932.

9 Li YN, Zhou LX, Qiang XH, et al. Effect of continuous blood purification and thymosin alphal on the cellular immunity in patients with severe sepsis: a prospective, randomized, controlled clinical trial. Zhongguo Wei Zhong Bing Ji Jiu Yi Xue, 2009, 21(3):139-142.

10 Orozco H, Arch J, Medina-Franco H, et al. Molgramostim(GM-CSF) associated with antibiotic treatment in nontraumatic abdominal sepsis: a randomized, double-blind, placebo-controlled clinical trial. Arch Surg, 2006, 141(2):150-153. 